\title{
Quantum calculation of Coulomb reorientation and near-barrier fusion
}

\author{
C. Simenel ${ }^{* \dagger}$, M. Bender ${ }^{* \dagger}$, Ph. Chomaz ${ }^{* *}$, T. Duguet ${ }^{\dagger *}$ and G. de France** \\ ${ }^{*}$ DSM/DAPNIA, CEA SACLAY, F-91191 Gif-sur-Yvette, France \\ ${ }^{\dagger} N S C L, M S U$, East Lansing, Michigan 48824, USA \\ ${ }^{* *}$ GANIL, B.P. 55027, F-14076 CAEN Cedex 5, France \\ $¥$ Physics and Astronomy Department, MSU, East Lansing, Michigan 48824, USA
}

\begin{abstract}
We investigate the role of deformation on the fusion probability around the barrier using the Time-Dependent Hartree-Fock theory with a full Skyrme force. We obtain a distribution of fusion probabilities around the nominal barrier due to the different contributions of the various orientations of the deformed nucleus at the touching point. It is also shown that the long range Coulomb reorientation reduces the fusion probability around the barrier.
\end{abstract}

\section{INTRODUCTION}

Fusion of massive nuclei has recently drawn a lot of interest, especially at energies around the fusion barrier generated by the competition between the Coulomb and nuclear interactions. In this energy domain, the reaction mechanisms may depend strongly on the structure of the collision partners. The proper description of near-barrier fusion is thus a challenging N-body quantum dynamical problem involving the competition between various reaction channels. For example, the coupling between the internal degrees of freedom and the relative motion may generate a fusion barrier distribution [1]. Such couplings are needed to reproduce the sub-barrier fusion [2].

One of the internal degrees of freedom which can strongly affect the fusion is the static deformation [3, 4]. First, the fusion probability depends on the orientation of the deformed nucleus at the touching point. Second, a reorientation can occur under the torque produced by the long-range Coulomb force [5, 6, 7, 8]. Such a reorientation is a consequence of the excitation of rotational states. It induces an anisotropy in the orientation distribution, thus modifying the near-barrier fusion [9].

In this work we study the fusion of a spherical and a prolate deformed nucleus within the Time-Dependent Hartree-Fock (TDHF) theory. We first show the effect of the orientation at the touching point on the fusion probability. Then we include the long range Coulomb excitation of rotational states and study its effect on fusion. The results give a useful interpretation of full coupling channels calculations. 


\section{TIME-DEPENDENT HARTREE-FOCK THEORY}

Let us first recall briefly some aspects of TDHF theory and of its numerical applications to nuclear collisions. TDHF is a mean field quantum dynamical theory [10, 11, 12] . It describes the evolution of occupied single particle wave functions in the mean field generated by all the particles. The total wave function of the system is constrained to be a Slater determinant at any time which assures an exact treatment of the Pauli principle during the dynamics. All standard applications of TDHF neglect pairing correlations so far. Like all mean-field methods, TDHF is best suited to desctibe average values of one-body operators. Such quantities are determined from the one-body density matrix $\hat{\rho}=\sum_{n=1}^{N}\left|\varphi_{n}\right\rangle\left\langle\varphi_{n}\right|$ where $\left|\varphi_{n}\right\rangle$ denotes an occupied single particle state. In TDHF, its evolution is determined by a Liouville-von Neumann equation, $i \hbar \partial_{t} \hat{\rho}=[\hat{h}(\rho), \hat{\rho}]$ where $\hat{h}(\rho)$ is the mean-field Hamiltonian.

The great advantage of TDHF is that it treats the static properties and the dynamics of nuclei within the same formalism, i.e. using the same effective interaction (usually of the Skyrme type [13]). The initial state is obtained through static Hartree-Fock (HF) calculations which are known to reproduce rather well nuclear binding energies and deformations. TDHF can be used in two ways to describe nuclear reactions:

- A single nucleus is evolved in an external field [14], simulating for instance the Coulomb field of the collision partner [7].

- The evolution of two nuclei, initially with a zero overlap, is treated in the same box with a single Slater determinant [15, 16].

The first case is well suited for the description of inelastic scattering, like Coulomb excitation of vibrational and rotational states. The second case is used for more violent collisions like deep-inelastic and fusion reactions. In such cases, the lack of a collision term in TDHF might be a drawback. At low energy, however, the fusion is mainly driven by the one-body dissipation because the Pauli blocking prevents nucleon-nucleon collisions. The system fuses mainly by transfering relative motion into internal excitation via one-body mechanisms well treated by TDHF.

Another important advantage of TDHF concerning its application to near-barrier reaction studies is that it contains implicitely all types of couplings between the relative motion and internal degrees of freedom whereas in coupling channels calculations one has to include them explicitely according to physical intuition which is not always straightforward for complex mechanisms. The only condition in TDHF is that the symmetries corresponding to the internal degrees of freedom of interest are relaxed. This is now the case with the latest TDHF codes in 3 dimensions (3D) which use a full Skyrme force [17, 18]. However, TDHF gives only classical trajectories for the time-evolution and expectation values of one-body observables. In particular, TDHF does not include tunneling of the global wave function.

We use the TDHF code built by P. Bonche and coworkers [17] using a Skyrme functional [13]. This code computes the evolution of each occupied single-particle wave function in a 3D box assuming one symmetry plane. The step size of the network is $0.8 \mathrm{fm}$ and the step time $0.45 \mathrm{fm} / \mathrm{c}$. We use the SLy4d parametrization [17] of the Skyrme force which is a variant of the SLy4 one specifically designed for TDHF calculations. 


\section{FUSION WITH A DEFORMED NUCLEUS}

\section{Effect of the static deformation}

Many nuclei exhibit static deformation, that is well described by mean-field calculations. Static deformation breaks the rotational invariance of the Slater determinant, which introduces an intrinsic frame of the nucleus. TDHF calculations of nuclear collisions, however, are performed in the laboratory frame, and one is left with an ambiguity concertning the relative orientation of the deformed nuclei. This is a critical point, because different orientations might ultimately lead to different reaction paths.

To illustrate this point we consider central collisions of a prolate deformed ${ }^{24} \mathrm{Mg}$ $\left(\beta_{2}=0.4\right)$ with a spherical ${ }^{208} \mathrm{~Pb}$. For symmetry reasons, the reaction mechanism will depend only on the energy and the angle between the deformation axis and the collision axis noted $\varphi$. Fig. 1 shows the time evolution of the density for two different initial orientations. We see that with an initial orientation $\varphi=0^{\circ}$ the nuclei fuse whereas with $\varphi=37.5^{\circ}$ the two fragments separate after a deep-inelastic collision.

The technic used to overcome the ambiguity of the initial orientation is based on two prescriptions [7, 8]:

1. It is necessary to assume an initial distribution of orientations.

2. Interferences between different orientations are neglected. Then each Slater determinant evolves in its own mean field.

Let us first assume an isotropic distribution of the orientations at the initial time, corresponding to a distance $D=20 \mathrm{fm}$ between the two centers of mass. This means that the ${ }^{24} \mathrm{Mg}$ is supposed to be initially in its $0^{+}$ground state and that all kind of long range Coulomb excitations are neglected up to this distance. Then, using the above prescriptions we get the fusion probability

$$
P_{f u s}(E)=\frac{1}{2} \int_{0}^{\pi} \mathrm{d} \varphi \sin \varphi P_{f u s}(E, \varphi)
$$

where $P_{f u s}(E, \varphi)=0$ or 1 . The solid line in Fig.2-a shows the resulting fusion probability as function of the center of mass energy. Below $93 \mathrm{MeV}$ no orientation leads to fusion and above $99 \mathrm{MeV}$, all of them fuse. Between these two values, the higher the energy, the more orientations lead to fusion. As shown in Fig. 1 configurations with small $\varphi$ are the first to fuse, even below the nominal barrier which would correspond to a spherical

${ }^{24} \mathrm{Mg}$ case (dotted line). To conclude, sub-barrier fusion is described in TDHF through couplings between static deformation and relative motion.

\section{Long range Coulomb reorientation}

As a consequence of numerical limitations, actual TDHF calculations for collisions are performed in relatively small boxes and are started with internuclear distances of a few Fermi. However, the Coulomb interaction starts playing a role much earlier in the reaction process [19]. It is weaker than the nuclear interaction, but integrated over a long 


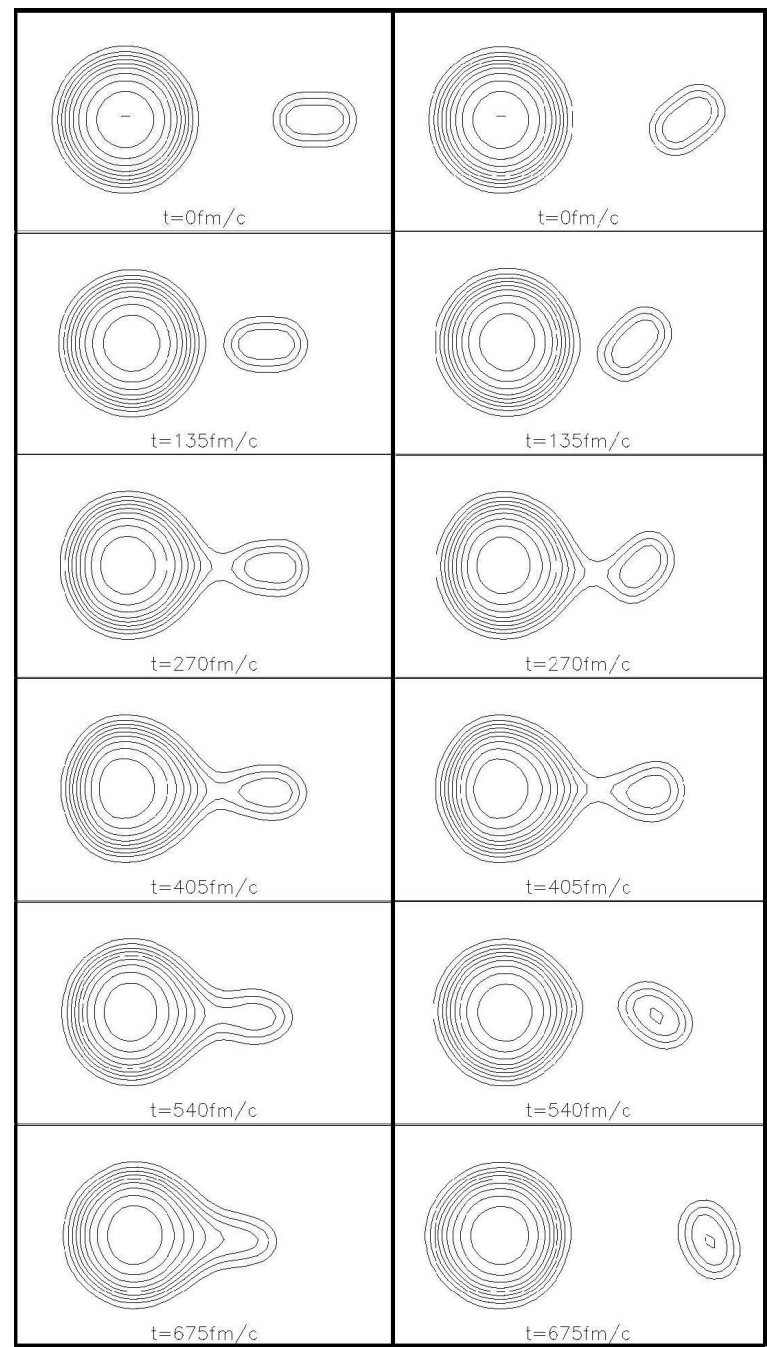

FIGURE 1. Density plots of head-on ${ }^{208} \mathrm{~Pb}+{ }^{24} \mathrm{Mg}$ collisions at $E_{C M}=95 \mathrm{MeV}$ with an initial orientation at $20 \mathrm{fm}$ of $0^{\circ}$ (left) and $37.5^{\circ}$ (right). The time step between each figure is $135 \mathrm{fm} / \mathrm{c}$.
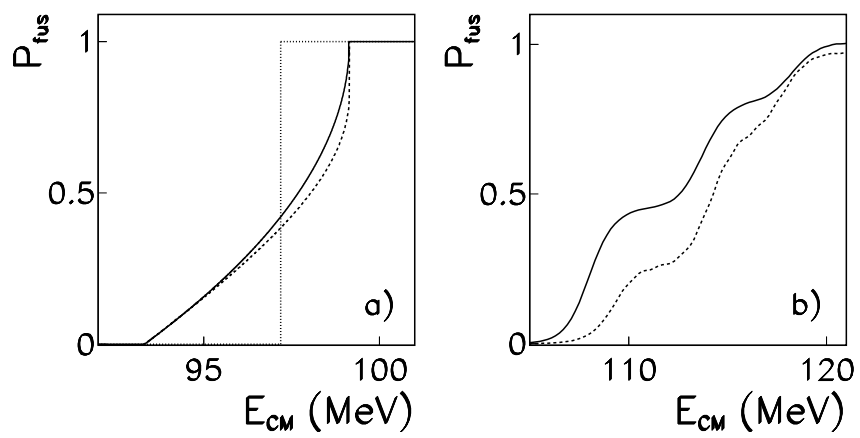

FIGURE 2. Fusion probability for a head-on collision or "penetrability" of ${ }^{208} \mathrm{~Pb}+{ }^{24} \mathrm{Mg}$ as a function of the c.m. energy. a) TDHF results. Isotropic distribution of the initial orientations is supposed at $20 \mathrm{fm}$ (solid line) and at $220 \mathrm{fm}$ (dashed line). Step function expected in case of a spherical ${ }^{24} \mathrm{Mg}$ (dotted line). b) CCFULL results without (solid line) and with (dashed line) Coulomb excitation. 
time it may induce a polarization, and then modify the reaction mechanism [7, 20]. Long range Coulomb reorientation has been studied in Ref. [7] with TDHF. The results have been interpreted using the classical formalism [19, 21] where the motion of a deformed rigid projectile is considered in the Coulomb field of the target. An important conclusion of this work is that the reorientation, although being the result of a Coulomb effect, depends neither on the charges nor on the energy. Let us illustrate this phenomenon with a simple example. Consider a system at time $t$ formed by a deformed projectile at the distance $D(t)$ from the target. Increasing artificially the charge of one of the nuclei at this time has two effects. First, the Coulomb interaction increases and then the torque applied on the deformed nucleus should increase too. On the other hand, the distance $D(t)$ between the projectile and the target is larger because of the stronger Coulomb repulsion between the centers of mass. The latter effect leads to a decrease of the effective torque at time $t$ and both effects overall cancel exactly. One is left with a charge independent reorientation. The same argument applies for the energy.

To study the effect of reorientation on fusion we calculate the reorientation in the approach phase between $D=220 \mathrm{fm}$ and $20 \mathrm{fm}$ with TDHF using the technic described in Ref. [7]. Assuming an isotropic distribution of orientations at $220 \mathrm{fm}$ we get a new distribution at $20 \mathrm{fm}$ which includes the reorientation coming from long range Coulomb excitation. The new fusion probability distribution (dashed line in Fig. 2-a) is obtained with two additional assumptions:

- The rotational speed of the ${ }^{24} \mathrm{Mg}$ is neglected at the initial time of the TDHF calculation (corresponding to $D=20 \mathrm{fm}$ ), i.e. only a static reorientation is considered.

- The effect of the excitation energy on the relative motion is neglected, i.e. we assume a Rutherford trajectory before $D=20 \mathrm{fm}$.

We observe in Fig. 2-a a fusion hindrance up to $20 \%$ which is due to higher weights on orientations leading to compact configurations $\left(\varphi \sim 90^{\circ}\right.$ at the touching point $)$ because of the reorientation [7, 20].

The previous study is helpful to interpret coupling channels results. Calculations on the same system have been performed with the code CCFULL [22] including coupling to the five first excited states of ${ }^{24} \mathrm{Mg}$ rotational band. The fusion probability, or "penetrability" of the fusion barrier is given by the relation $P_{f u s}=\frac{\mathrm{d}(\sigma E)}{\mathrm{d} E} \pi R_{B}^{2}$ where $R_{B}=11.49 \mathrm{fm}$ is the barrier position. Fig. 2. b shows the fusion probability obtained from CCFULL including nuclear (solid line) and nuclear+Coulomb (dashed line) couplings. As with TDHF, an hindrance of the fusion due to Coulomb couplings is observed. However the shape of TDHF and CCFULL distributions are quite different. This is due to the fact that quantum mechanical effects are missing in TDHF. This point out the importance of improving the theory. It is also striking to see that TDHF "misses" the nominal barrier by about $15 \%$. TDHF is known to overestimate the fusion cross sections. One possible issue might be the time odd terms in the Skyrme energy functional. Their importance on fusion have been stressed recently [18, 23]. 


\section{CONCLUSION}

To summarize, we performed a TDHF study of near-barrier fusion between a spherical and a deformed nucleus. The calculations show that, around the barrier, different orientations lead to different reaction path. Considering all possible orientations leads to a distribution of fusion probabilities interpreted as an effect of the coupling between the static deformation and the relative motion. We then included the long range Coulomb coupling which induces a charge and energy independent reorientation of the deformed nucleus. The effect of the reorientation is to hinder the near-barrier fusion. Finally the TDHF study have been used to interpret coupling channels calculations with the code CCFULL which show also an hindrance of near-barrier fusion due to Coulomb couplings. We also note some drawbacks of TDHF which, in one hand, underestimates the fusion barrier, and, in the other hand, miss important quantum effects.

\section{ACKNOWLEDGMENTS}

We warmly thank Paul Bonche for providing his TDHF code. This work has been partially supported by NSCL, Michigan State University and the National Science Foundation under the grant PHY-0456903.

\section{REFERENCES}

1. C.H. Dasso, S. Landowne and A. Winther, Nucl. Phys. A 405, 381 (1983).

2. M. Dasgupta, D.J. Hinde, N. Rowley and A.M. Stefanini, Annu. Rev. Nucl. Part. Sci. 48, 401 (1998).

3. P.W. Riesenfeldt and T.D. Thomas, Phys. Rev. C 2, 711 (1970).

4. A.S. Jensen and C.Y. Wong, Phys. Rev. C 1, 1321 (1970).

5. H. Holm, W. Scheid and W. Greiner, Phys. Lett. B 29, 473 (1969).

6. L. Wilets, E. Guth and J.S. Tenn, Phys. Rev. 156, 1349 (1967).

7. C. Simenel, Ph. Chomaz and G. de France, Phys. Rev. Lett. 93, 102701 (2004).

8. A.S. Umar and V.E. Oberacker, nucl-th/0604010

9. L.T. Baby et al., Phys. Rev. C 62, 014603 (2000).

10. D.R. Hartree, Proc. Camb. Phil. Soc. 24, 89 (1928).

11. V.A. Fock, Z. Phys. 61, 126 (1930).

12. P.A.M. Dirac, Proc. Camb. Phil. Soc. 26, 376 (1930).

13. T. Skyrme, Phil. Mag. 1, 1043 (1956).

14. D. Vautherin and D.M. Brink, Phys. Rev. C 5, 626 (1972).

15. P. Bonche, S. Koonin and J.W. Negele, Phys. Rev. C 13, 1226 (1976).

16. J.W. Negele, Rev. Mod. Phys. 54, 913 (1982).

17. K.-H. Kim, T. Otsuka and P. Bonche, J. Phys. G 23, 1267 (1997).

18. A.S. Umar and V.E. Oberacker, nucl-th/060338.

19. K. Alder and A. Winther, electromagnetic excitation (north-holland/american elsevier, 1978).

20. N. Rowley, N. Grar and K. Hagino, Phys. Lett. B 632, 243 (2006).

21. R.A. Broglia and A. Winther, Heavy-Ion Reactions (Addison-Wesley, New York, 1991).

22. K. Hagino, N. Rowley and A.T. Kruppa, Comp. Phys. Com. 123, 143 (1999).

23. J.A. Maruhn, P.-G. Reinhard, P.D. Stevenson and M.R. Strayer, nucl-th/0604070 